\title{
Analyse économique du syndicalisme agricole. Une typologie comparative à partir des défaillances de marché
}

Economic analysis of agricultural trade unionism. (A comparative typology based on market failures)

\section{Jean Vercherand}

\section{(2) OpenEdition}

\section{Journals}

Édition électronique

URL : http://journals.openedition.org/economierurale/867

DOI : 10.4000/economierurale. 867

ISSN : 2105-2581

\section{Éditeur}

Société Française d'Économie Rurale (SFER)

Édition imprimée

Date de publication : 10 août 2009

Pagination : 93-109

ISSN : 0013-0559

Référence électronique

Jean Vercherand, «Analyse économique du syndicalisme agricole. Une typologie comparative à partir des défaillances de marché », Économie rurale [En ligne], 312 | Juillet-août 2009, mis en ligne le 05 juillet 2011, consulté le 01 mai 2019. URL : http://journals.openedition.org/economierurale/867 ; DOI : 10.4000/economierurale.867 


\section{Analyse économique du syndicalisme agricole Une typologie comparative à partir des défaillances de marché}

Jean VERCHERAND • Inra, Laboratoire de recherche sur les innovations socio-techniques et organisationnelles en agriculture (Listo), Dijon

\section{Introduction}

$\mathbf{E}^{\mathrm{n}}$ France comme dans la plupart des pays, 'objet du syndicalisme est «l'étude et la défense des intérêts » professionnels (Reynaud, 1975) ${ }^{1}$. Il est donc très général. Aussi, trouve-t-on des syndicats dans de nombreuses activités économiques et catégories socioprofessionnelles : salariés, employeurs, professions indépendantes, agriculteurs, etc. De même, le mutualisme et le coopératisme ont été et peuvent être encore une composante interne du mouvement syndical ${ }^{2}$. Cependant, cette « défense des intérêts » a des limites. Si elle conduisait les acteurs à se constituer en cartel, par exemple en rationnant l'offre pour hausser les prix de vente, leur action tomberait immédiatement sous le joug des lois contre les ententes (ou anti-trust). Ainsi, le principal syndicat agricole français et d'autres organisations de la filière viande ont été lourdement sanctionnés par la Commission européenne, en avril 2003, pour entente illicite sur le marché de la viande bovine. On peut donc faire l'hypothèse que le législateur n'accepte et ne légalise l'action des syndicats que si celle-ci concoure à rendre les échanges marchands plus efficients (en tout cas, pas moins) : en précisant les normes et les conditions y contribuant, ou en palliant

1. Selon l'article 3 de la loi du 21 mars 1884 qui, en France, les légalise : «Les syndicats professionnels ont exclusivement pour objet l'étude et la défense des intérêts économiques, industriels, commerciaux et agricoles ».

2. Le Droit a généralement reconnu que les syndicats pouvaient s'adonner à des activités d'achat, vente, revente et location au profit de leurs adhérents à condition de ne pas réaliser de bénéfices. les défaillances éventuelles des marchés. $A$ contrario, on peut considérer que si les échanges étaient parfaitement et constamment efficients, la défense des intérêts par les syndicats serait sans utilité.

Or, de ce point de vue, l'histoire du syndicalisme - dont l'origine est avant tout ouvrière - apparaît très paradoxale. À la fin du XVIII ${ }^{\mathrm{e}}$ siècle, les premiers pays industriels ont proclamé les grands principes du libéralisme économique. Les grèves et ce qu'on appellera plus tard les syndicats sont prohibés car considérés comme des entraves à la libre concurrence sur le marché du travail. Or, face aux problèmes majeurs qui apparaissent alors sur ce marché, tous les pays démocratiques reviennent sur ces interdits, généralement à la fin du XIX ${ }^{\mathrm{e}}$ siècle (dès 1824-25 au Royaume Uni). Les ÉtatsUnis sont, à cet égard, un cas plus éclairant. Les syndicats n'y ont jamais été prohibés car participant des libertés publiques fondamentales, en revanche, leurs méthodes d'actions étaient régulièrement condamnées par les tribunaux en vertu de la législation antitrust. Ce n'est qu'avec la loi Clayton, en 1914, que l'application de cette législation est supprimée pour le marché du travail (Parias, 1963).

Pourquoi donc, les comportements d'entente ont-ils été acceptés pour ce marché alors qu'ils restent prohibés ailleurs ? Qu'est-ce que ce marché du travail avait de si particulier pour que le législateur fasse une exception quant au rôle que peuvent y exercer les syndicats? Les juristes, de même que les historiens et les sociologues, spécialistes des problèmes du travail, ont répondu : c'est parce que les salariés sont pour la plupart dans une situation d'infériorité de 
rapport de force, de dépendance économique vis-à-vis des employeurs, que le législateur leur a concédé le droit de se coaliser. Cette réponse entraîne à son tour deux questions :

- Comment caractériser, du point de vue de l'analyse économique, cette dépendance économique des salariés à l'égard des employeurs, qui est à l'origine des syndicats et de leur légalisation mais aussi, qui justifie la spécificité du syndicalisme salarié par rapport aux autres syndicalismes ?

- En quoi le syndicalisme agricole se rapproche-t-il ou se différencie-t-il du syndicalisme salarié quant aux caractéristiques des rapports marchands qu'entretiennent les agriculteurs avec leur environnement ? Autrement dit, parmi les nombreuses situations de marché conflictuelles dans l'agriculture, existe-il des états de dépendance économique qui se rapprochent de celui qui prévaut sur le marché du travail ? Et si c'est le cas, de quelle manière ces états de dépendance se manifestent-ils ?

Cette approche comparative, partant du marché du travail, est indispensable car, outre l'origine ouvrière de la loi sur les syndicats, on rencontre dans le syndicalisme agricole, historiquement, tous les courants idéologiques et politiques qui entendaient au $\mathrm{XIX}^{\mathrm{e}}$ et $\mathrm{XX}^{\mathrm{e}}$ siècles résoudre la question sociale (c'est-à-dire résoudre les problèmes que posait la nature très conflictuelle du marché du travail) ce qui, en revanche, n'est pas le cas du syndicalisme patronal, ni de celui des professions indépendantes (Barral, 1968 ; Duby et Wallon, 1976 ; Hervieu et Lagrave, 1992 ; Lynch, 2002 ; Vercherand, 1994).

L'objet de cet article est donc de spécifier et de mettre en perspective, du point de vue de l'analyse économique, les raisons d'être du syndicalisme agricole et des actions qu'il conduit, par comparaison avec les autres syndicalismes, en particulier salarié. Pour ce faire, nous partirons du principe suivant : si les rapports d'échange étaient constamment optimaux, le syndicalisme, en tant que défense des intérêts, serait inutile, donc inexistant. Aussi, sur la base des rapports d'échange problématiques, voire conflictuels, que l'on peut rencontrer historiquement entre les acteurs dans l'agriculture, nous nous attacherons à analyser et à caractériser les défaillances ou les distorsions des marchés qui peuvent en être la cause. Par symétrie, nous chercherons à inventorier les actions conduites pour rendre les échanges plus efficients.

Ensuite, se pose la question de l'ordre de classement des différentes activités des syndicats professionnels du point de vue de l'analyse économique (sachant que le rôle des syndicats n'est pas purement revendicatif, ni dans l'agriculture et autres professions indépendantes, ni même chez les salariés). Pour cela, nous partirons du constat suivant : tout agent économique entretient nécessairement des relations d'échange binaires avec les autres agents : comme offreur et comme demandeur (également comme émetteur et récepteur d'effets externes). Dans ces relations d'échange, certaines sont problématiques, voire antagoniques, ce qui traduit des défaillances ou des distorsions par rapport à une situation idéale de marchés efficients. Cela conduit généralement les agents concernés par un même problème à s'organiser pour :

- s'adresser à la partie adverse afin de le résoudre ;

- s'adresser à la puissance publique afin d'aider à le résoudre ;

- agir en interne afin de pallier par euxmêmes la difficulté rencontrée (par exemple en s'entraidant).

On aboutit ainsi à trois grands domaines d'intervention - que l'on trouve peu ou prou dans tous les syndicalismes - à l'égard : des autres agents, interlocuteurs des échanges ; de l'État; des adhérents.

Ajoutons, concernant ce troisième domaine, que des contradictions internes, liées par exemple à des concurrences exacerbées, 
peuvent surgir. Elles peuvent conduire les syndicats à intervenir pour les résoudre sinon il y a un risque d'éclatement de ceux-ci.

Cependant cette typologie présente des difficultés particulières pour l'agriculture en raison de la très grande hétérogénéité économique et sociale de ce secteur, et par le fait que les rapports d'échange problématiques y sont nombreux, aussi bien en amont (situations de demandeurs) qu'en aval (situations d'offreurs) des agriculteurs. C'est l'occasion de préciser que, dans cette approche typologique, nous partirons des exploitants agricoles ${ }^{3}$. Aussi, les syndicalismes des salariés agricoles, des propriétaires bailleurs, des divers négociants et industriels en lien avec l'agriculture, etc., n'apparaîtront, explicitement ou implicitement, qu'en réciproque, c'est-à-dire comme interlocuteurs de celui des exploitants. Cela dit, dans cet article, il ne sera pas possible d'être exhaustif dans l'analyse des actions conduites par le syndicalisme agricole. C'est avant tout sur ses raisons d'être fondamentales que l'on mettra l'accent, c'est-à-dire sur les défaillances ou distorsions de marché existantes (ou ayant existé).

Avant de développer notre typologie analytique des raisons d'être du syndicalisme agricole et de ses actions, nous présenterons rapidement, dans une section préliminaire, celles du syndicalisme salarié et de ses actions à titre de référence et de comparaison.

3. Les termes «syndicalisme agricole» sont ambigus pour le profane s'agissant d'un milieu très hétérogène historiquement, allant des domestiques de ferme aux grands propriétaires fonciers. Ainsi, on ne saurait parler de « syndicalisme industriel » sans préciser s'il s'agit des patrons ou des ouvriers. Cependant, ces termes sont consacrés par l'usage et c'est la raison pour laquelle nous les conservons. Sans doute est-ce là le legs du projet corporatiste des catholiques sociaux, principaux promoteurs des « syndicats agricoles » qui devaient regrouper toute la hiérarchie du monde agricole mais qui, en réalité, n'ont rassemblé très essentiellement que les exploitants et, après la Seconde Guerre mondiale, exclusivement ceux-ci.

\section{L'origine du syndicalisme L'asymétrie du marché du travail}

Les raisons d'être du syndicalisme salarié sont, du point de vue de la théorie économique, très discutées (Paquet et al, 2004 ; Jardin, 2000). En fait, cela ne fait que refléter les controverses, en amont, concernant la représentation du marché du travail.

Examinons d'abord rapidement les faits (Parias, 1963). On sait qu'en 1791, les décrets d'Allarde ont aboli en France les corporations qui avaient régi pendant sept siècles les rapports de concurrence et les rapports de travail au sein de chaque métier. Ensuite, la loi Le Chapelier a interdit, en raison des grands principes libéraux, toute coalition (ou entente) sur le marché du travail : les grèves et ce qu'on nommera les syndicats sont illégaux. Or, ce marché, qui semblait satisfaire, a priori, aux conditions d'une concurrence pure et parfaite, se révèle profondément conflictuel, avec des revendications récurrentes pour hausser les salaires mais aussi pour réduire la durée du travail (Perrot, 1973). En dépit des interdits, des grèves éclatent, lesquelles peuvent dégénérer en affrontements, émeutes, voire insurrections quand les forces de l'ordre et la justice interviennent pour faire respecter le Droit. L'histoire du marché du travail est parsemée d'épisodes violents et tragiques dans tous les pays industriels, ce qui n'est pas banal tout de même quand on est économiste et qu'on s'intéresse aux rapports marchands... Parallèlement, émerge une série de courants idéologiques, - critiquant le libéralisme économique pour les uns, le libéralisme économique et politique pour les autres, et qui entendent résoudre cette question sociale. Cela va des différents courants socialistes et anarchistes, jusqu'au néocorporatisme, en passant par des courants intermédiaires ne remettant en cause ni la propriété privée, ni les principaux acquis de la Révolution, mais jugent nécessaire de protéger les travailleurs. Le communisme a été un échec 
total sur le plan économique et une tragédie humaine épouvantable mais il n'est pas tombé du ciel. Il se voulait être une bonne réponse à une vraie question, la question sociale, c'est-à-dire aux défaillances majeures qui affectaient le marché du travail.

Finalement, dans les pays démocratiques, s'est imposé l'édification d'un Droit du travail - toujours plus ou moins contesté par nombre d'économistes - qui a commencé par une limitation de la durée du travail (c'est-à-dire de l'offre), puis par l'acceptation des comportements d'entente (ou de coalition) des employés (Le Goff, 2004). Ainsi, le travail est le seul marché dont les conflits (les grèves) donnent lieu à un recensement par les statistiques officielles et, de ce fait, à des comparaisons internationales ; Le seul, également, où les comportements de monopole sont légaux. Tout cela, encore une fois, est ni banal, ni anodin.

Or, ni la nouvelle microéconomie du travail, ni de façon générale les différentes théories contemporaines qui ont pu être avancées et qui ont fait l'objet de synthèses ( $c f$. entre autres : Cahuc et Zylberberg, 1996, 2004 ; Gazier, 1992 ; Perrot, 1995 ; Redor, 1999 ; Smith, 2003), ne permettent véritablement de rendre compte de ces faits, de comprendre cette énorme singularité du marché du travail. D'ailleurs, la multiplicité des modèles théoriques proposés en constitue un aveu implicite. Cela fait du marché du travail, l'un des domaines les plus controversés de toute l'analyse économique.

C'est chez les juristes du travail que l'on peut trouver une réponse qui semble beaucoup plus congruente avec les faits (Pélissier et al., 2006). Une réponse, en réalité, qui avait été esquissée et développée chez certains classiques, respectivement par Smith, Sismondi et Marx pour ne prendre que les plus connus : Le marché du travail est caractérisé par une inégalité de rapport de force, due à la base à une inégalité (ou asymétrie) dans les dotations en facteurs de production ${ }^{4}$. L'employé ne possède généralement que sa force de travail à offrir sur le marché pour vivre, tandis que l'employeur possède également sa propre force de travail mais, en plus, les moyens matériels de production (le capital). Il en résulte une situation de dépendance économique du premier à l'égard du second que les juristes ont définie ainsi : "État d'un travailleur, salarié ou non, vis-à-vis de la personne qui l'emploie, lorsqu'il tire du travail qu'il exécute pour cette personne ses principaux moyens d'existence ${ }^{5}$. Sur le plan de la représentation économique du marché du travail, cela signifie, non seulement que : - les salariés n'optimisent pas leurs préférences (leurs choix) en matière d'offre de travail, mais que les employeurs sont en mesure de peser, au gré de leurs intérêts et du rapport de force dont ils disposent (limité précisément par le Droit), sur le volume de travail fourni, c'est-à-dire sur sa durée et sur son intensité (les cadences); et donc, de peser sur les salaires. D'ailleurs, le Droit a toujours reconnu que la fixation de la durée du travail est une prérogative patronale dont un salarié ne peut se soustraire sans dommage ${ }^{6}$.

4. À ne pas confondre avec l'hypothèse d'asymétrie de l'information, ni avec celle de monopsone ou d'oligopsone.

5. Définition en droit du travail. La perte d'autonomie économique des travailleurs résulte, avant la révolution industrielle, des processus de concentration foncière qui ont chassé de leurs terres de nombreux paysans, puis, la grande industrie triomphant, de la ruine de couches de plus en plus étendues de producteurs indépendants qui n'ont pu résister à la concurrence de la production de masse à bas prix.

6. Un salarié (ie un travailleur dépendant) peut toujours demander à travailler à temps partiel ou à faire des heures supplémentaires mais la décision finale appartient à son employeur, contrairement au travailleur indépendant parfaitement libre de ses choix en ce domaine. 
En d'autres termes, si on part de la croix de Saint André qui schématise un équilibre de marché, pour le travail, nous avons la branche descendante (la courbe de demande) mais pas de branche ascendante (la courbe d'offre) ; cette dernière doit être remplacée par une plage d'offre dans laquelle les employeurs peuvent puiser. L'offre n'est pas autonome de la demande mais subordonnée à cette dernière ${ }^{7}$. C'est là, toute la singularité du marché du travail par rapport aux autres marchés. Cela dit, on ne développera pas ici comment s'opèrent les équilibres à court et à long terme d'un tel marché ${ }^{8}$.

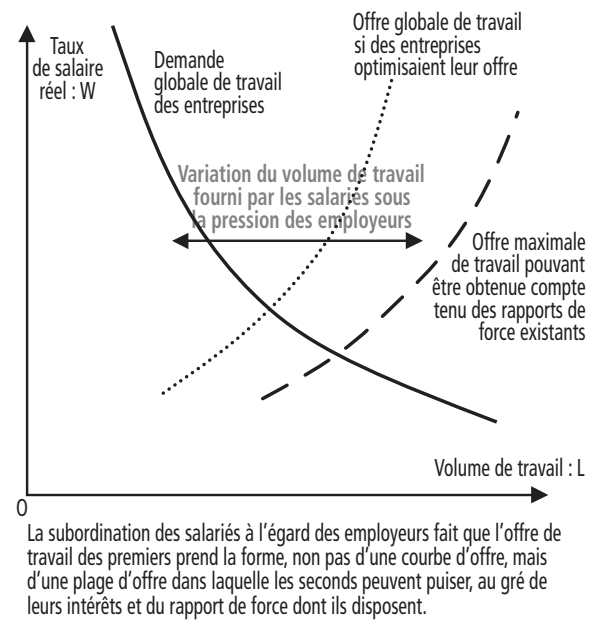

Dès lors, sur la base de cette thèse, empruntée aux juristes et à certains économistes classiques, cette histoire sociale devient intelligible du point de vue de l'analyse économique, en particulier la raison profonde du syndicalisme salarié et, plus

7. Des modèles théoriques peuvent faire état de relations de subordination des salariés à l'égard des employeurs mais ce n'est pas pour envisager une situation où les employeurs seraient en mesure de peser sur la durée et les cadences de travail. Dans ces modèles, la relation de subordination porte sur le caractère vérifiable ou non du travail fourni par les salariés ( $c f$. : Cahuc et Zylberberg, 1996, chapitres 4 et 5 , p. 229-354).

8. Voir pour cela Vercherand (2006) ou, pour une présentation plus concise, Vercherand (2004), chapitres $5, \S 1$ et 12 . largement, de ce que l'on appelle le mouvement ouvrier.

Si on prend le raisonnement typologique présenté en introduction, l'action du syndicalisme ouvrier (et plus largement salarié) a porté historiquement (et porte toujours) sur les domaines suivants (Andolfatto et Labbé, 2006 ; Dreyfus, 2001 ; Mouriaux, 2005 ; Sagnes, 1994).

Des actions revendicatives, comme offreurs (employés), auprès des employeurs, au niveau de l'entreprise, puis, à mesure que le dialogue social s'est construit, au niveau de la branche et de l'ensemble de celles-ci, depuis le bassin d'emploi jusqu'à l'échelon national ; mais aussi des actions revendicatives, comme demandeurs (consommateurs), à l'encontre des pratiques jugées déloyales de la part d'entreprises de production et de distribution des biens de consommation. (Le mouvement consumériste trouve son origine dans le syndicalisme ouvrier au XIX ${ }^{\mathrm{e}}$ siècle et, aujourd'hui, la plupart des centrales syndicales disposent de leurs propres associations de consommateurs). Cette seconde activité est, bien évidemment, beaucoup moins importante que la première.

- Des actions revendicatives auprès de l'État pour obtenir son arbitrage, puis son intervention protectrice, enfin pour faire évoluer le Droit quand celui-ci s'est peu à peu édifié (Droit du travail, Droit social, Droit de la consommation, fiscalité, etc.) ; Mais aussi des actions de cogestion avec l'État et le patronat des services publics de sécurité sociale, d'assurance chômage, et autres organismes qui participent du dialogue social.

- Des actions de service auprès des adhérents avec la création de sociétés de secours mutuel ${ }^{9}$, de coopératives de

9. En France, le mouvement mutualiste s'est séparé du syndicalisme ouvrier à la suite des décisions politiques prises au début du Second empire. En revanche, dans la plupart des pays industriels, les deux sont restés unis (Dreyfus, op. cit.). 
consommation, d'activités d'éducation populaire, de tourisme social, de conseils juridiques, etc. ; des actions aussi pour aplanir les concurrences : entre travailleurs immigrés et nationaux, salariés du public et du privé, entre qualifications, etc.

- Dans le syndicalisme patronal, comme dans celui des professions libérales et indépendantes (pour partie employeuses de maind'œuvre), on trouve en parallèle ou en réciproque ces mêmes trois domaines, bien que certains d'entre eux y tiennent une place plus réduite (Brizay, 1975 ; Fraboulet, 2007 ; Reynaud, op. cit.).

\section{Actions du syndicalisme agricole envers les acteurs des échanges}

\section{Envers les fournisseurs (offreurs) de facteurs de production agricole}

\section{La main-d'ouvre agricole}

On ne traitera pas de la nature du rapport d'échange à l'œuvre ici puisqu'il correspond à ce qui vient d'être présenté. On soulignera simplement quelques spécificités du travail salarié en agriculture. Il a longtemps concerné une main-d'œuvre en déclin, isolée et dispersée dans de nombreuses unités de production au collectif de travail réduit, souvent sous l'emprise des employeurs. Toutefois, depuis près d'une vingtaine d'années, le salariat dans la production agricole augmente. Il représente aujourd'hui près de $30 \%$ du travail fourni dans les exploitations professionnelles.

\section{Les propriétaires fonciers bailleurs}

Avant la Seconde Guerre mondiale et plus encore au XIX ${ }^{\mathrm{e}}$ siècle, les exploitants fermiers et métayers étaient, en beaucoup de régions françaises, en situation de grande dépendance à l'égard des propriétaires bailleurs et fortement pressurés par eux. La durée des baux était courte (1 à 3 ans en général, rarement au-delà) et leur renouvellement soumis au bon vouloir du propriétaire.
L'asymétrie de rapport de force était patente, surtout entre le métayer qui ne possédait quasiment que sa force de travail à employer pour faire vivre sa famille et le propriétaire qui possédait en plus de sa propre force de travail - qu'il pouvait toujours employer à sa guise - la terre et en partie le capital d'exploitation. L'analogie avec le marché du travail est donc grande du fait de cette relation de dépendance et on le verra plus loin - de l'existence d'un droit spécifique pour ce marché de location du sol. Du point de vue de l'analyse économique, cette asymétrie de rapport de force vient d'une demande qui était très abondante, provenant de nombreux paysans en concurrence, non possesseurs ou insuffisamment possesseurs de la terre, alors que l'offre est parfaitement inélastique : la terre est un bien rare, offert par la nature en quantité donnée (invariable), et approprié de façon inégalitaire. Toutefois ici, les propriétaires n'étaient pas en mesure de peser directement sur le volume de travail fourni - comme dans le marché du travail - mais sur le tribut foncier payé par le tenancier. Cette concurrence était potentiellement plus forte en métayage où pouvait se porter demandeuse - par phénomène de vases communicants - une maind'œuvre composée de journaliers et de domestiques, largement dépourvue de capital puisque celui-ci était apporté, au moins en partie, par le propriétaire en sus de la terre et des bâtiments. Inversement, là où le capital requis de la part du fermier était plus élevé (dans les grandes exploitations), la concurrence y était moindre et les grands fermiers en position plus favorable. Autre différence avec le marché du travail, le tenancier conservait la direction de la production : le fermier, très largement et le métayer, en partie ; le propriétaire n'ayant qu'un rôle faible ou partiel.

Révélateur de cette dépendance, les métayers ont été tentés de s'engager (voire se sont engagés) en certaines régions, non pas dans les syndicats agricoles « clas- 
siques » mais à la CGT, avant et après la Première Guerre mondiale. De manière générale, les petits fermiers et surtout les métayers ont constitué - avec les ouvriers agricoles - le principal vivier dans lequel les forces de gauche et d'extrême gauche ont puisé leurs militants et sympathisants dans les campagnes. Cette mobilisation a débouché sur la promulgation, en 19451946, du «Statut du fermage et du métayage », dont l'objet était de protéger celui qui travaille la terre.

\section{Le commerce et les industries agroalimentaires d'approvisionnement}

Les premiers syndicats agricoles et, avec eux, les premières coopératives, ont été créés dans le but d'approvisionner les agriculteurs, au meilleur rapport qualité/prix, en engrais et autres facteurs de production et, ainsi, les protéger contre les fraudes de certains négociants quant à la qualité de ces produits. Cette réaction collective venait du fait qu'individuellement, les agriculteurs n'avaient guère les moyens de vérifier la qualité des produits industriels nouveaux qu'ils achetaient. Certes, à la longue, ils pouvaient acquérir une connaissance plus précise de celle-ci mais, dans un contexte d'innovation technique, même à rythme modéré, il leur était difficile d'évaluer en continu ce rapport qualité/prix. D'ailleurs, il pouvait en être de même pour les commerçants finaux des villages : la fraude ne se réalisant pas forcément au stade ultime du circuit commercial. La confiance qui avait pu se forger au cours du temps entre ces commerçants et les agriculteurs ne suffisait plus. Bref, on est typiquement en présence de ce que l'on appelle une asymétrie de l'information.

Des situations d'oligopoles dans l'agrochimie (engrais, produits phytosanitaires...) et le machinisme ont pu être dénoncées. Cependant, l'attitude syndicale à ce propos relevait plutôt d'une rhétorique anticapitaliste - sauf peut-être dans le secteur des engrais ${ }^{10}$ - car aucun conflit tangible ne peut, à notre connaissance, être signalé. Les développements théoriques sur l'oligopole, la concurrence indirecte et potentielle, les marchés contestables montrent que la rente occasionnée par ces situations oligopolistiques est finalement peu avérée.

\section{Envers les acheteurs (demandeurs) des produits agricoles}

\section{Le commerce et les industries \\ agroalimentaires}

Plus fréquents furent les tensions et les conflits entre les agriculteurs et le secteur aval qui collecte, conditionne, transforme et commercialise leur production. Ceux-ci recouvrent un grand nombre de cas de figure.

On rencontre également, comme précédemment, des situations d'asymétrie de l'information même si celles-ci sont de nature quelque peu différente. C'est l'exemple bien connu de l'éleveur isolé et sous informé jadis face au maquignon, fin évaluateur des animaux et parfaitement informé de l'état du marché et des prix qui se pratiquent. L'agriculteur pouvait être conduit à céder sa production à un prix sous évalué par rapport à celui du marché. Cependant, ce cas de figure était moins le fait de négociants importants, depuis longtemps en place, connus et reconnus. Il provenait généralement de petits ou de nouveaux intervenants qui parvenaient à tenir ou cherchaient à s'imposer en faisant des « coups », en sollicitant directement chez eux et nouant des relations interpersonnelles étroites, des agriculteurs qui n'avaient pas toujours la possibilité de se déplacer sur les lieux de marché.

10. De la fin de la Première Guerre mondiale jusqu'aux années 70, l'État a exercé en France, un véritable monopole sur la production de potasse, dénoncé par d'autres fabricants d'engrais et par certaines organisations agricoles comme coûteux pour les agriculteurs et les contribuables. $C f$. Gardinier (1974). 
En outre, sur ces marchés, les acheteurs, moins nombreux et se connaissant davantage que les agriculteurs entre eux, ont pu être soupçonnés de nouer des ententes pour faire pression sur les prix. Cependant, on sait que ce type de pratique est instable et reste relativement ponctuel et limité. D'ailleurs, si on prend toujours le cas de la viande, la coopération ne s'est imposée que très tardivement, à partir des années 1960 grâce au soutien des pouvoirs publics, notamment par les lois d'orientation de 1960 et 1962 qui encouragent l'organisation des marchés avec les groupements de producteurs. Cela laisse à penser que le système précédent qui mobilisait de multiples intermédiaires, souvent dénoncé par les deux extrémités du circuit, les agriculteurs et les consommateurs, n'était pas si défaillant. En réalité, si le syndicalisme agricole a pu vilipender dans ses discours les pratiques de tromperie et d'ententes des maquignons c'était surtout dans une visée instrumentaliste : s'opposer à leur concurrence pour l'accès à la terre et convaincre les agriculteurs de s'engager dans les formes « organisées » de commercialisation avec les groupements de producteurs et les coopératives. Néanmoins, ces derniers ont contribué à rendre les marchés plus transparents en œuvrant pour imposer des bases plus objectives de paiement de la production agricole (selon le poids et la grille de classement des carcasses en viande).

Des cas d'oligopsone, voire de monopsone, ont pu être évoqués comme en production laitière où, en certaines régions, il n'existe qu'un seul collecteur. En réalité, les entreprises laitières n'ont jamais refusé du lait, c'est-à-dire restreint leur demande pour faire baisser le prix à la production, pour une raison simple : les mécanismes publics d'intervention prenaient automatiquement en charge les excédents éventuels de production. Les écarts de prix entre régions sont relativement faibles et s'expliquent principalement par des différences dans les frais de collecte, dans les valorisations du lait (produits AOC) et dans l'efficacité des entreprises. De toute façon, les producteurs ont toujours la possibilité de prendre en charge la collecte par la création de coopératives, puis de revendre le lait au transformateur le plus offrant et le plus fiable. Une telle stratégie a pu être mise en œuvre avant et après la Seconde Guerre mondiale par le syndicalisme en certaines régions (exemple : le Bassin lyonnais). Cependant, il s'agissait là d'une stratégie principalement préventive, pour éviter les comportements de monopsone et la défaillance éventuelle d'un transformateur et, on le verra plus loin, pour mieux traiter les situations d'excédents de production. Là également, si le syndicalisme a pu s'en prendre aux «trusts laitiers » dans les années 1970, c'était davantage dans le cadre d'une rhétorique anticapitaliste que pour dénoncer des comportements réels de monopsone.

Les trois cas qui viennent d'être présentés, asymétrie de l'information, ententes et monopsone (ou oligopsone), sont finalement peu avérés en aval de la production agricole.

Plus tangibles mais en même temps plus imprécis sont les reproches faits aux industries agroalimentaires et à la grande distribution de dominer les agriculteurs et de multiplier les exigences à leur égard. Des manifestations parfois «musclées » ont pu être conduites à l'encontre de certaines grandes surfaces... Le nombre des grandes centrales d'achat par lesquelles se concentrent les flux commerciaux étant réduit, le grand commerce est soupçonné en permanence de pressurer en amont ses fournisseurs. Cependant, pour qu'il puisse faire pression sur les prix à la fourniture, il faudrait qu'il restreigne ses achats en amont et, du même coup, les reventes en aval en direction des consommateurs. Or, il existe toujours des circuits parallèles de commercialisation, effectifs ou potentiels, qui interdisent ce genre de pratiques. En réalité, l'asymétrie de rapport de force vient ici d'une tendance récurrente à la surproduction 
alors que la demande alimentaire est généralement très inélastique (rigide). La surproduction peut avoir plusieurs causes : des circonstances climatiques favorables (maraîchage, arboriculture, ...) ; une structure particulière de l'offre (coûts et temps de réponse de la production) engendrant une alternance entre pléthore et pénurie (cycle du porc, ...) ; une dynamique de progrès technique forte alors que la main-d'œuvre agricole est peu mobile professionnellement et géographiquement (lait, ...) ; etc. Dans ces conditions, il est logique que la rigidité de la demande se retourne contre les producteurs quand leur offre est excessive. En revanche, quand, pour une raison ou pour une autre, l'offre se raréfie sur les marchés, l'asymétrie s'inverse. Pensons aux éleveurs de porcs quand le cycle des prix est au plus haut, au retournement du marché du lait et des produits laitiers en 2007, ou à la situation des agriculteurs pendant la période de pénurie alimentaire due à la Seconde Guerre mondiale... Là également, ce n'est, ni le grand commerce, ni l'industrie alimentaire qui pèse sur les volumes offerts. Contrairement à la relation salariale.

Ce sont ces situations de surproduction ponctuelles qui ont motivé le développement de la coopération en aval de la production agricole, bien plus que l'asymétrie d'information ou les soupçons d'entente ou de comportements de monopsone des acteurs privés. D'ailleurs, cet essor de la coopération aval a été fortement encouragé par les pouvoirs publics, en périodes de crises, afin de servir de relais aux politiques publiques d'intervention sur les marchés qui seront examinées plus loin. Ces politiques ont été complétées en 1974 et 75 par la mise en place «d'interprofessions » dont l'objet principal est de favoriser les accords contractuels entre les acteurs d'une filière ce qui, outre la régularisation des marchés, permet d'économiser sur les coûts de transactions.

Cela dit, cette organisation interprofessionnelle n'est pas assimilable à des ententes au préjudice des consommateurs car les groupements de producteurs et les coopératives restent en concurrence et, par ailleurs, tous les acteurs d'une filière (agriculteurs, entreprises agroalimentaires, commerce de gros ou de détail) n'adhèrent pas forcément à ces formes organisées. Elle n'est donc pas en mesure d'influencer durablement l'offre des agriculteurs, ni les prix à la production. Dans les secteurs où l'intervention publique pour régulariser les marchés est faible ou inexistante (viandes, fruits, légumes, etc.), pour hausser les prix à la production, il faudrait que tous les agriculteurs de l'Europe se liguent pour limiter leur offre ; Ou bien, à l'intérieur d'un seul pays, par exemple la France, que tous les acteurs d'une filière, y compris la grande distribution, conviennent de limiter l'offre des agriculteurs et de ne pas recourir aux importations pour approvisionner le marché intérieur ${ }^{11}$. Dans les secteurs où l'intervention publique est forte comme le lait, une entente (en faveur des producteurs) peut négliger l'offre et ne porter que sur les prix. Dès lors, dans un pays donné, il faudrait que tous les acteurs de la filière, y compris la grande distribution, s'accordent sur un

11. Ce sont pour des pratiques assez proches que la FNSEA et d'autres organisations de la filière viande ont été sanctionnées en avril 2003 d'une très grosse amende par la Commission européenne pour entente illicite lors de la seconde crise de la vache folle. Si l'entente est avérée, son impact sur les prix, en amont et en aval, semble avoir été négligeable. Quoi qu'il en soit, la question centrale que pose cette sanction est de savoir s'il est légitime de traiter de la même manière, d'un côté un syndicat qui cherche à défendre, même maladroitement, l'existence même de ses mandants dont les revenus sont déjà, en moyenne et en temps normal, parmi les plus faibles de l'agriculture et, de l'autre, de grandes entreprises qui s'entendent pour réaliser des surprofits (ou profits purs au sens de la théorie) ? Peut-on assimiler des travailleurs indépendants qui cherchent à compenser leur position momentanée d'extrême infériorité dans la chaîne des relations économiques, à des entreprises qui font abus de position dominante ? Sur le plan théorique, nous nous trouvons ici aux frontières du Droit commercial (dont relève l'abus de position dominante) et du Droit du travail. 
prix minimum à la production et s'engagent impérativement à ne pas recourir à des importations intra-européennes pour approvisionner le marché intérieur. On est très loin de cela et ce serait surestimer considérablement la puissance du syndicalisme agricole de croire qu'il puisse développer une telle stratégie. Le niveau et l'évolution des revenus des agriculteurs, dans les interprofessions qui pourraient être soupçonnées d'ententes, en sont la meilleure preuve...

\section{Les particuliers consommateurs}

On ne s'étendra pas sur ce domaine d'intervention - mentionné pour mémoire dans la mesure où les relations de vente directe des agriculteurs aux consommateurs ont été peu problématiques historiquement. En revanche, le syndicalisme agricole a souvent été conduit à intervenir ou à se concerter avec les administrations publiques quant à la législation qui entoure ce mode de commercialisation ( $c f$. infra, la section «Interventions par rapport aux normes d'une concurrence saine et loyale »).

\section{Envers les intégrateurs}

Au cours des années 1960, se sont développés en élevage hors sol des systèmes intégrés de production. Des entreprises agroalimentaires, privées ou coopératives, fournissaient à des agriculteurs les animaux à élever, l'aliment et souvent le protocole technique de production, puis elles reprenaient les animaux finis pour les commercialiser. Les agriculteurs, qui devaient réaliser eux-mêmes l'investissement dans les bâtiments et équipements de l'élevage, étaient rémunérés sur la différence entre le prix de rachat de leur production et le coût des fournitures en amont. Généralement, ces contrats d'intégration étaient souscrits par de petits agriculteurs qui cherchaient dans ces productions intensives à développer leur activité et leurs revenus mais disposaient de capitaux limités. Certes, dans ce type de contrats, il y a un gradient très vaste de cas concrets quant à la nature précise de la relation qui lie les deux parties... Or, à l'occasion d'un retournement des marchés en aval, ou bien à la suite d'une dégradation des performances technico-économiques de l'élevage - parfois en raison de la fourniture d'un aliment de mauvaise qualité -, des éleveurs se sont retrouvés, non seulement avec leur travail impayé, mais aussi dans l'impossibilité de rembourser leurs emprunts liés aux investissements. Il s'en est suivi une longue lutte syndicale durant les années 1970, conduite par le mouvement «paysan-travailleur » qui voyait dans ces formes d'intégration la préfiguration de la subordination de l'agriculture dans le mode de production capitaliste. L'agriculteur devenait un ouvrier à façon supportant tous les risques techniques et économiques de son activité. Cette lutte syndicale a débouché sur une évolution du Droit en matière d'intégration afin de mieux protéger les personnes.

\section{Envers les tiers émetteurs ou récepteurs d'effets externes}

Ces effets externes relèvent, contrairement aux situations précédentes, de marchés virtuels dans la mesure où les titres de propriété des « services » en question ne sont pas très bien identifiés, ni surtout reconnus. Ils concernent : soit des dommages occasionnés par l'activité agricole sur des tiers (pollutions, odeurs, bruits, assèchement de cours d'eau causé par l'irrigation, etc.) ; soit des dommages que des usagers de l'espace agricole (promeneurs, etc.) peuvent provoquer sur l'activité agricole (barrières de parcs laissées ouvertes, cultures détruites, etc.). Quand les émetteurs de ces effets externes négatifs sont identifiés, divers marchandages sont envisageables : négociations ou poursuites judiciaires en réparation, etc. Quand l'identification est difficile, des négociations peuvent s'engager entre organisations représentatives des intérêts en présence pour résoudre au mieux les différends. Le syndicalisme agricole peut être sollicité dans les deux cas comme 
partie prenante de la médiation. De même, il peut l'être par des collectivités locales ou des associations diverses dans le but de conforter des effets externes positifs ou de développer des services collectifs intentionnels provenant des agriculteurs (ex. : production non intentionnelle ou intentionnelle par les agriculteurs d'un paysage ou d'un espace de promenade apprécié du public).

\section{Action du syndicalisme agricole envers l'État}

\section{Sur des questions générales}

Interventions contre l'ouverture au libre-échange

La protection de la production nationale contre la concurrence étrangère a été l'une des premières et des plus constantes revendications du syndicalisme agricole ${ }^{12}$. On connaît la loi des avantages comparatifs (1817) de Ricardo qui légitime le libreéchange. Cependant, un certain nombre d'arguments, incitant à la prudence en la matière, sont reconnus par les économistes : notamment pour en réguler les effets sur la main-d'œuvre afin qu'elle puisse se reconvertir et passer, de proche en proche, des secteurs concurrencés vers ceux qui seront bénéficiaires d'une ouverture des échanges. Ce qui pose problème, là aussi, c'est le fait que la mobilité des facteurs de production (travail et capital) n'est pas parfaite mais visqueuse - sinon, il n'y aurait pas d'opposition au libre-échange. Ce point est particulièrement important dans l'agriculture où les écarts de productivité sont très grands à travers le monde et alors que, dans de nombreux pays en développement,

12. Il en est de même pour les premières organisations patronales qui apparaissent au XIX ${ }^{\mathrm{e}}$ siècle et dont le souci constant jusqu'au lendemain de la Seconde Guerre mondiale sera de dénoncer la concurrence étrangère. Ainsi le Conseil national du patronat français (CNPF) s'est opposé à la création de la CECA en 1951 (mais non à la CEE en 1957 et au marché commun qu'elle institue) (Brizay, 1975). la population active agricole est très élevée. Dans ces conditions, une ouverture rapide au libre-échange aurait des effets déstabilisateurs extrêmement brutaux.

\section{Interventions par rapport au Droit du travail} On ne s'étendra pas sur ce point déjà abordé dans la section préliminaire. Comme l'ensemble du patronat, celui de l'agriculture s'est opposé aux avancées du Droit en faveur des salariés. Arguant de la spécificité du travail dans l'agriculture (dépendances vis-àvis des cycles culturaux, des conditions climatiques, de la lumière naturelle du jour...; rémunération en nature des salariés avec le gîte et le couvert fournis par l'exploitant ; etc.), il fut, des décennies durant, le plus rétrograde : vive opposition dans l'entre-deux guerres aux lois sur les assurances sociales et sur la durée du travail (journée de 8 heures, semaine de 40 heures). Aussi, le Droit appliqué aux salariés de la production agricole fut longtemps en retard sur celui des autres salariés.

\section{Interventions par rapport aux normes}

d'une concurrence saine et loyale

Les économistes conviennent que les marchés concurrentiels - quand ils sont possibles - sont préférables à ceux qui ne le sont pas (hormis le cas particulier des biens sous tutelle), toutes choses restant égales par ailleurs. Cependant un marché concurrentiel ne se met pas forcément en place spontanément. Comme pour toute compétition - puisque c'en est une - il suppose des règles de jeu et un arbitre (l'État de droit) pour les faire respecter. Sinon, tous les coups seraient permis et le jeu (le marché) dégénérerait rapidement. La théorie économique énonce un certain nombre de conditions pour qu'un marché fonctionne de façon efficace pour la société, c'est-à-dire pour que la concurrence y soit saine et loyale.

Les pouvoirs publics ont été conduits à préciser ces conditions par voie législative et réglementaire, en particulier en matière de transparence de marché. Parallèlement, les 
organisations syndicales (ou ce qui en tenait lieu) ont été sollicitées pour avis (ou ont pris position) sur ces questions, puis, pour leur application concrète. Dans l'agriculture, cela s'applique à de très nombreux domaines. Dans toutes les productions agricoles, animales et végétales, on trouve la même mise en place de critères sanitaires et qualitatifs de définitions des produits, aussi objectivables que possible. La notion de qualité, elle-même, est très complexe. Outre les aspects sanitaires et de richesse du produit, il y a également ceux relatifs au goût, aux résidus extérieurs qu'il peut contenir, aux conditions de production avec la mise en place de procédures de certification, à la traçabilité, etc. On connaît les débats en cours sur la question des Organismes génétiquement modifiés (OGM) ou des hormones...

\section{Interventions par rapport à la prise en compte des effets externes}

Plus une société se développe, plus les effets externes prennent une place grandissante dans les choix individuels et collectifs (élasticité-revenu $>1$ ). Les agriculteurs sont en première ligne sur ces questions dans la mesure où ils gèrent directement une grande partie de l'espace (les deux tiers du territoire national en France). Leurs façons de produire inter-réagissent donc fortement, de façon positive ou négative, sur l'environnement - la ressource en eau et les paysages en particulier - mais aussi de manière très générale sur la perception qu'en a le reste de la société : pensons à la question du bien-être animal... Il en résulte une intervention grandissante des politiques publiques sur ces effets externes qui interpelle directement le syndicalisme agricole et l'oblige à se situer.

\section{Sur des questions agricoles}

Intervention par rapport au Droit du foncier Cette intervention a concerné d'abord et surtout le «Statut du fermage et du métayage »-que l'on retrouve dans tous les pays développés où ces modes de fairevaloir du sol sont importants. Il s'est appliqué au prix d'un long combat juridique et de terrain du syndicalisme des preneurs, face à une propriété foncière regroupée, à partir de 1945, dans la Fédération nationale de la propriété agricole (FNPA). Le principal résultat de ce statut a été de casser radicalement la concurrence entre agriculteurs face à la propriété bailleresse, en sécurisant très fortement le fermier en place sur la terre qu'il cultive, en limitant le droit de reprise par le propriétaire et en imposant un barème de prix pour le montant des loyers. Il en a découlé, d'un côté, un affaiblissement de la rente foncière prélevée par la propriété foncière et, de l'autre, un dynamisme d'investissement et des gains de productivité considérables dans l'agriculture. Ce statut est un bon exemple d'intervention publique qui, comme le Droit du travail, limite la concurrence sur un marché très particulier pour atteindre une plus grande efficacité économique globale.

D'autres interventions publiques ont suivi, à l'égard desquelles le syndicalisme s'est situé : en particulier la «politique des structures » - réclamée par une partie du syndicalisme agricole et mise en place dans les années 1960 - qui visait à pallier la forte viscosité de la mobilité professionnelle de la main-d'œuvre agricole à une période de modernisation intense de l'agriculture et de concurrence effrénée pour l'accès à la terre.

\section{Intervention face à la régularisation des} marchés agricoles

L'intervention publique sur les marchés agricoles est très ancienne. C'est même une constante historique : le pouvoir politique s'est toujours préoccupé au plus haut degré de « la question des subsistances ». Des différents règlements de l'Antiquité à « la police des grains » sous l'Ancien régime, de « l'échelle mobile » de la Restauration à la PAC d'aujourd'hui, une suite longue et tâtonnante de dispositifs réglementaires a 
encadré la production et la commercialisation des denrées alimentaires, en particulier des « bleds », base principale de l'alimentation pendant des siècles.

La raison profonde, bien connue des économistes ruraux, est la forte instabilité des marchés agricoles. Ceux-ci combinent, pour la plupart, une demande très inélastique (rigide) et une offre qui peut fluctuer fortement. D'un côté, la demande est très rigide et non différable dans le temps pour la simple raison que les gens veulent manger tous les jours, à leur faim, ni plus, ni moins. La capacité des estomacs reste invariable ! Aussi, il suffit d'une petite variation des quantités offertes (par rapport à une situation moyenne) pour qu'elle engendre une variation de prix en sens contraire de grande ampleur. Gregory King avait constaté dès 1696 que les producteurs agricoles réalisaient collectivement leurs meilleures recettes, non lorsque la récolte était abondante mais lorsqu'elle était médiocre... De l'autre côté, l'offre peut fluctuer fortement en raison des aléas naturels mais aussi de choix surréactifs des agriculteurs. Les aléas naturels ne sont pas seulement climatiques (gel, sécheresse, grêle, ...) mais concernent aussi les maladies végétales et animales (épizooties), les insectes et les animaux (les organismes classés «nuisibles ») qui peuvent détruire les récoltes et les troupeaux. Quant aux choix surréactifs des agriculteurs, ils proviennent du fait que l'offre agricole est relativement élastique à moyen terme : même s'ils sont dépendants d'assolements cycliques permettant une gestion durable du potentiel productif de leurs sols, les agriculteurs peuvent à la marge réorienter une part significative de leurs surfaces vers telle ou telle culture selon que les prix de vente (espérés) sont élevés ou bas. Parallèlement, il peut exister un décalage de temps important entre la décision de produire (ou ne pas produire) et l'arrivée effective de la production sur le marché, en raison des cycles biologiques. Il en résulte un fonctionnement chaotique du marché. La pléthore peut succéder à la pénurie et l'effondrement des prix à leur flambée ; et réciproquement : un problème majeur pour les populations que le pouvoir politique a toujours cherché à résoudre historiquement, tant bien que mal... Cette instabilité a longtemps conduit la paysannerie à des comportements d'une extrême prudence, routiniers voire malthusiens, que des générations de promoteurs du «progrès » se sont efforcées de combattre. L'analyse économique montre que l'offre est sous optimale en situation d'incertitude (Boussard et Delorme, 2007). La théorie montre aussi que ces fluctuations entraînent une perte de bien-être pour la société, au préjudice des consommateurs quand la demande est plus inélastique que l'offre (ce qui est souvent le cas à moyen terme pour la production agricole $)^{13}$.

Après avoir longtemps essayé de résoudre ces problèmes en réglementant le commerce des denrées et en jouant sur l'ouverture des frontières, l'État s'est résolu, lors de la grande crise des années 1930, à intervenir directement sur les marchés pour les régulariser. Face à cela, le syndicalisme a souvent fait preuve d'une grande ambivalence. La revendication vigoureuse d'une intervention de l'État en cas de crise a pu côtoyer une grande défiance à l'égard de celle-ci. En fait, par rapport à une situation idéale d'équilibre et de stabilité, toute défaillance de marché engendre des perdants et des gagnants. Globalement parlant, les agriculteurs ne sont pas les perdants des fluctuations des prix agricoles ( $c f$. la note 13). Cependant, leur condition de gagnant ou de perdant varie : selon les marchés (tous n'ont pas la même âpreté concurrentielle, ni

13. Ces pertes de bien-être peuvent être appréhendées à partir d'un cobweb convergent vers un cycle permanent (le cobweb est divergent à l'intérieur de la zone de fluctuation des prix - la demande y est plus élastique que l'offre - et convergent à l'extérieur). On peut alors montrer aisément que ces fluctuations sont coûteuses pour la société, avec pertes pour les consommateurs mais gains pour les producteurs sur chaque cycle. 
la même instabilité), selon les périodes (les hauts prix succèdent aux bas prix), et surtout selon les individus (certains résistent mieux aux crises ou sont plus chanceux que d'autres). Les agriculteurs peuvent donc hésiter entre un revenu modeste mais régulier et un revenu plus élevé en moyenne mais irrégulier.

Quoi qu'il en soit, cette régulation des marchés a contribué à un dynamisme exceptionnel de l'offre après la Seconde Guerre mondiale : sécurisés face à l'aléa du prix, les agriculteurs ont investi massivement dans le progrès technique et la production. Parallèlement, cette régulation s'est accompagnée de la mise en place de divers organismes, privés et publics, gérés ou cogérés avec l'État, par des représentants syndicaux ${ }^{14}$. Ceci a conduit le syndicalisme à interpeller constamment les décideurs politiques pour hausser les prix institutionnels. Pourtant, paradoxalement, cela n'a pas engendré une sur-rémunération des agriculteurs. Au contraire, sommes-nous porté à dire. Si on rapporte historiquement la part que représente l'agriculture dans la valeur ajoutée totale du pays (le PIB) à celle que représentent les agriculteurs dans la population active totale on arrive à un ratio de l'ordre de 1 à 2 depuis la fin de la Seconde Guerre mondiale. En d'autres termes, la rémunération des facteurs de production dans l'agriculture est, en dépit du soutien des marchés, relativement faible en moyenne. D'ailleurs, c'est en raison du fait que leurs efforts de productivité ne leur semblaient pas payés en retour qu'une partie du courant syndical

14. Cette « cogestion » a été très décriée. Pourtant, on la retrouve aussi dans le syndicalisme salarié : cogestion de la Sécurité sociale, de l'assurance chômage, des comités d'entreprise, etc. En fait, la critique visait surtout l'élaboration très corporatiste de la politique agricole qui s'était instaurée, dans les années 1970, avec la «Conférence annuelle » : le principal syndicat agricole négociait directement cette politique avec le gouvernement et court-circuitait le parlement et toutes les composantes de la société intéressées par celle-ci.
« moderniste » des jeunes agriculteurs s'est radicalisée dans une critique anticapitaliste à la fin des années 1960.

La théorie économique enseigne que lorsque les débouchés croissent moins rapidement que la productivité des facteurs et que la mobilité de ces derniers est visqueuse, leur sous-rémunération est alors forte. On ne développera pas davantage ce point.

\section{Actions du syndicalisme agricole envers ses adhérents}

La coopération et la mutualité (y compris en matière de crédit) ont été, au début, une composante entière de l'activité syndicale de « défense des intérêts », tout comme les nombreux autres services individuels et collectifs dispensés aux agriculteurs : information, formation, enseignement, conseils techniques, économiques, juridiques, fiscaux, etc. La coopération et la mutualité se sont développées, à l'origine, dans le but de réduire les facteurs d'incertitude qui pesaient sur les agriculteurs, en matière : de calamités (assurances mutuelles contre la mortalité du bétail, la grêle, l'incendie, ... secours divers), de financement, d'accident corporels, de santé et de vieillesse, de marché... Elles ont constituées également un outil essentiel de la politique agricole de l'État. Leur autonomie actuelle, en France, vis-à-vis du syndicalisme, résulte de décisions politiques prises entre 1940 et 46 en matière de structuration professionnelle de l'agriculture. En revanche, dans de nombreux pays européens, les fonctions syndicales, coopératives et mutualistes sont regroupées et intégrées (Hervieu et Lagrave, 1992). De même, aujourd'hui en France, le conseil technique est dispensé par les Chambres d'agriculture (un type d'organisme qu'on ne trouve pas dans tous les pays européens), à la suite de décisions politiques prises dans les années 1950 et 60 . Cependant, le syndicalisme a généralement veillé à se réserver la dispense de certains services, considérés comme stratégiques 
pour s'attacher l'adhésion des agriculteurs. C'est le cas aujourd'hui du conseil fiscal et juridique mais aussi, depuis l'origine du syndicalisme, de la presse professionnelle qui est un outil indispensable pour les agriculteurs. Cette dispense de services est le principal facteur explicatif des taux d'adhésion syndicale, chez les agriculteurs, dans les autres professions ou chez les salariés (Rosanvallon, 1998).

\section{Conclusion}

La levée du délit de coalition sur le marché du travail fonde, à la fin du XIX ${ }^{\mathrm{e}}$ siècle, la reconnaissance du syndicalisme dans la plupart des pays industriels. Il s'agissait alors de la première étape d'un processus législatif qui visait à compenser l'état d'infériorité (d'asymétrie de rapport de force) de l'un des contractants (l'ouvrier) par rapport à l'autre (l'employeur) et, ainsi, à pacifier les relations de travail.

Or, dans les défaillances de marché (envisagées dans le corpus néoclassique), les inégalités dans les dotations en facteurs de production (et en patrimoine) - qui font que certains acteurs ne disposent d'aucune autonomie économique et se trouvent de fait sous la dépendance d'autres acteurs mieux lotis dans les relations d'échange n'ont jamais été envisagées ${ }^{15}$. En revanche, dans le corpus classique, ce rapport de dépendance a été développé - à la suite de l'ébauche d'Adam Smith - par plusieurs économistes hétérodoxes, en particulier

15. Pourtant, parmi les conditions nécessaires à la réalisation d'un équilibre général, figure l'existence de dotations initiales de survie permettant à chaque ménage de survivre sans faire d'échange. Cette condition a pour but d'éviter que des ménages ne disparaissent au cas où la vente de leur dotation initiale (par exemple leur force de travail) ne leur permette même pas de survivre tant le prix de vente de celle-ci est bas. Or, précisément, quand la survie des ménages (au sens propre comme au sens figuré) est engagée, des formes de dépendances ne peuvent que s'instaurer biaisant fondamentalement la loyauté de l'échange.
Sismondi et Marx. Pourtant, c'est bien là, dans le présent comme dans le passé, la condition de la plupart des salariés face aux détenteurs du capital, mais aussi des métayers et petits fermiers face à la propriété bailleresse, de certains agriculteurs peu dotés et souscrivant un contrat d'intégration déséquilibré avec des IAA, ou encore d'agriculteurs en difficultés se plaçant sous la coupe d'un interlocuteur économique (fournisseur ou acquéreur) jouant le rôle de créancier...

Les situations d'oligopole ou d'oligopsone, d'information imparfaite, d'offre surabondante quand la demande est inélastique, entraînent également une certaine asymétrie de rapport de force. Cependant, elles n'engendrent pas une dépendance économique comparable aux cas précédents : les possibilités de choix alternatifs restent plus ouvertes. Autrement dit, dans les relations d'échange, il existe un gradient très vaste d'asymétries de rapport de force et de situations de dépendance pouvant en découler. D'ailleurs, la notion de dépendance économique se retrouve en Droit commercial et en Droit du travail mais dans des acceptions différentes, traduisant ainsi - très grossièrement - cette grande diversité d'asymétries de rapport de force. La montée du chômage de masse, au cours des 30 dernières années, a conduit les juristes du travail à s'interroger sur une extension du Droit du travail chez les travailleurs non salariés dans la mesure où des formes de dépendance économique proches de celle des salariés pouvaient s'y développer par capillarité (Lyon-Caen, 1990).

Face à ces asymétries de rapport de force, le syndicalisme (au sens très général de « défense des intérêts ») apparaît en démocratie, chez les agriculteurs et chez les salariés, comme un outil essentiel de défense d'acteurs en position d'infériorité, un outil de régulation sociale, mais aussi de propositions et contre-propositions politiques. S'ajoute, dans l'agriculture, dans 
les autres secteurs professionnels et chez les salariés, une fonction interlocutrice nécessaire des pouvoirs publics pour la définition et la mise en place des différentes normes qui concourent à un fonctionnement plus efficace d'une économie basée sur des échanges loyaux. Enfin, les services restent très nécessaires à une population d'agriculteurs, responsables des processus et des moyens de production, dispersés et isolés sur l'ensemble du territoire. Le syndicalisme peut s'investir dans leur diffusion afin de s'attacher les adhésions - limitant ainsi les comportements de « passager clandestin » à son action collective - et de gagner en force et en légitimité. Par le développement du mutualisme et du coopératisme, ce fut là sa plus belle réussite.

\section{Remerciements}

L'auteur remercie ses collègues du LISTO et les lecteurs anonymes pour l'intérêt de leurs observations sur une première version de ce texte.

\section{RÉFÉRENCES BIBLIOGRAPHIQUES}

Andolfatto D., Labbé D. (2006). Histoire des syndicats (1906-2006). Paris, Le Seuil. Barral P. (1968). Les agrariens français de Méline à Pisani. Paris, Armand Colin, Presses de la FNSP.

Boussard J.-M., Delorme H. (dir.) (2007). La régulation des marchés agricoles internationaux. Paris, L'Harmattan.

Brizay B. (1975). Le Patronat : histoire, structure, stratégie du CNPF. Paris, Le Seuil.

Cahuc P., Zylberberg A. (1996). Économie $d u$ travail, la formation des salaires et les déterminants du chômage. Bruxelles, De Boeck Université, réédition en anglais, Labor economics, 2004, Boston, The MIT Press.

Dreyfus M. (2001). Liberté, égalité, mutualité. Mutualisme et syndicalisme. 18521967. Paris, Éditions de l'Atelier.

Duby G. et Wallon A. (sous la dir.) (1976). Histoire de la France rurale, t. 2 : (dir.) Le Roy Ladurie E., t. 3 (dir.) Juillard E., t. 4 Gervais M., Jolivet M., Tavernier Y. Paris, Le Seuil.

Fraboulet D. (2007). Quand les patrons s'organisent. Stratégies et pratiques de l'UIMM (1901-1950). Villeneuve d'Ascq, Presses universitaires du Septentrion.

Gardinier L. (1974). La profession des engrais. Paris, La maison rustique.
Gazier B. (1992). Économie du travail et de l'emploi, Paris, $2^{\mathrm{e}}$ éd., Dalloz.

Hervieu B., Lagrave R.-M., (dir.) (1992). Les syndicats agricoles en Europe. Paris L'Harmattan.

Jardin E. (2000). L'analyse économique du syndicalisme, Ou les représentations économiques du syndicalisme : perspective historique, thèse de doctorat ès sciences économiques (dir. Caire G.), Nanterre, Janvier, Université Paris-X.

Le Goff J. (2004). Du silence à la parole : Une histoire du droit du travail des années 1830 à nos jours. Rennes, Presses universitaires.

Lynch E. (2002). Moissons rouges. Les socialistes français et la société paysanne durant l'entre-deux-guerres (1918-1940). Villeneuve d'Ascq, Presses universitaires du Septentrion.

Lyon-Caen G. (1990). Le Droit du travail non salarié. Paris, Sirey.

Mouriaux R. (2005). Le syndicalisme en France. Paris, PUF.

Paquet R., Tremblay J.-F., Gosselin E. (2004). Des théories du syndicalisme. Synthèse analytique et considérations contemporaines. In «Relations industrielles », Québec, vol. 59, n², Printemps.

Parias L.-H. (sous la dir.) (1963). Histoire générale du travail, t. 3 (dir.) Fohlen C., 
Bédarida F., t. 4 (dir.) Touraine A. Paris, Nouvelle Librairie de France.

Pélissier J., Supiot A., Jeammaud A. (2006).

Droit du travail, Paris, Dalloz, $23^{\mathrm{e}}$ éd.

Perrot A. Les nouvelles théories du marché du travail. (1995). La Découverte, Paris.

Perrot M. (1973). Les ouvriers en grève : France 1871-1890, Paris, Mouton, 2 t.

Redor D. (1999). Économie du travail et de l'emploi. Paris, Montchrestien.

Reynaud J.-D. (1975). Les syndicats en France. Paris, Le Seuil, 2 t.

Rosanvallon P. (1998). La question syndicale. Paris, Hachette.

Sagnes J. (dir.) (1994). Histoire du syndi- calisme dans le monde. Toulouse, Privat. Smith S. (2003). Labour economics. $2^{\text {sd }}$ ed., London, Routledge.

Vercherand J. (1994). Un siècle de syndicalisme agricole. La vie locale et nationale à travers le cas du département de la Loire. Publications de l'Université de Saint-Étienne.

Vercherand J. (2004). Économie politique. Une articulation entre la théorie néoclassique, Marx, Keynes et Schumpeter. Rennes, Presses universitaires.

Vercherand J. (2006). Le Travail. Un marché pas comme les autres. Rennes, Presses universitaires. 